\title{
Estimating the Annual Incidence of Abortions in Iran Applying a Network Scale-up Approach
}

\author{
Azam Rastegari ${ }^{1}$; Mohammad Reza Baneshi ${ }^{1}$; Saiedeh Haji-maghsoudi ${ }^{2}$; Nowzar Nakhaee \\ ${ }^{3}$; Mohammad Eslami ${ }^{4}$; Hossein Malekafzali ${ }^{5}$; Ali Akbar Haghdoost ${ }^{6,}$ \\ ${ }_{1}^{1}$ Research Center for Modeling in Health, Institute for Futures Studies in Health, Kerman University of Medical Sciences, Kerman, IR Iran \\ ${ }^{2}$ Social Determinant of Health Research Center, Institute for Futures Studies in Health, Kerman University of Medical Sciences, Kerman, IR Iran \\ ${ }_{3}^{3}$ Research Center for Health Services Management, Institute for Futures Studies in Health, Kerman University of Medical Sciences, Kerman, IR Iran \\ ${ }_{5}^{4}$ Department of Family and School Health, Ministry of Health and Medical Education, Tehran, IR Iran \\ 5 Department of Family and School Health, Ministry of Health and Medical Education, Tehran, IR Iran \\ 6 Department of Biostatistics and Epidemiology, School of Public Health, Tehran University of Medical Sciences, Tehran, IR Iran \\ ${ }^{*}$ Corresponding Author: Ali Akbar Haghdoost, Regional Knowledge Sciences, Tehran Hub for HIV/AIDS Surveillance, Institute for Futures Studies in Health, Kerman University of \\ Medical Sciences, P. O. BOX: 7619813159, Kerman, IR Iran. Tel/Fax:+98-3412263725, E-mail: ahaghdoost@kmu.ac.ir
}

Received: October 30, 2013; Revised: January 28, 2014; Accepted: March 11, 2014

\begin{abstract}
Background: Abortions are of major public health concern in developing countries. In settings in which abortion is highly prohibited, the direct interview is not a reliable method to estimate the abortion rate. The indirect estimation methods to measure the rate of abortion might overcome this dilemma; They are practical methods to estimate the size of the hidden group who do not agree to participate in a direct interview.

Objectives: The aim of this study was to explore the practicality of an indirect method for estimating the abortion rate, Known as Network Scale-up, and to provide an estimate about the episode of abortion with and without medical indications (AWMI+ and AWMI-) in Iran.

Materials and Methods: This cross-sectional study was conducted in 31 provinces of Iran in 2012. A random sample between 200 and 1000 was selected in each province by the multistage sampling method that $75 \%$ of the data were collected from the capital and $25 \%$ from one main city. We selected samples from urban people more than 18 years old (12960) and we asked them about the number of abortion in women they knew who had experienced the medical and non-medical abortions in the past year. A range for the transparency factor was estimated based on the expert opinion.

Results: The range of the transparency factors for AWMI+ and AWOMI- were 0.43-0.75 and 0.2-0.34, respectively. Regarding the AWMI+, our minimum and maximum estimations (per 1000 pregnancies) were 70.54 and 116.9, respectively. The corresponding figures for AWMIwere 93.18, and 148.7.

Conclusions: The frequency rates for AWMI+ and AWMI- were relatively high. Therefore, the system has to address to this hidden problem using the appropriate preventive policies.
\end{abstract}

Keywords:Abortion; Iran; Size Estimation; Network Scale-Up

\section{Background}

The frequency of abortion is one of the key indices in reproductive health. An unsafe abortion is a leading cause of maternal mortality and it contributes to $13 \%$ of maternal deaths worldwide. Each year, more than one third of pregnancies end in stillbirth and abortion, either spontaneous or induced. Nearly one of each 10 pregnancies is terminated by an unsafe abortion worldwide (1), and almost all unsafe abortions take place in developing countries.

For a deep exploration of this issue, the special attention to different types of abortion is required. Abortion could be divided into two types: induced and spontaneous, which may be differentiated by intention (2). Induced abortion per se could be performed in either safe or unsafe settings (1). An unsafe abortion is defined as "as a procedure for terminating an unintended pregnancy carried out either by persons lacking the necessary skills or in an environment that does not conform to minimal medical standards, or both" (3).

It is generally accepted that any intervention to prevent the unsafe abortion will ultimately depend on our information about the extent of the problem. In most Middle East and North Africa (MENA) countries, including Iran, induced abortion is legally restricted (4) (With a few exceptions). In Iran therapeutic abortion is allowed before 4 months of gestation only if the mother's life is in danger or in cases of sever fetal anomalies such as meningocele. Social and political issues constrain the epidemiologic studies related to abortion in any country, but this limitation is more evident in countries in which the induced abortion is legally prohibited, such as Iran (1).

It is important to mention that the estimation of abortion in Islamic countries such as Iran is more complex.

Copyright (C) 2014, Iranian Red Crescent Medical Journal; Published by Kowsar. This is an open-access article distributed under the terms of the Creative Commons Attribution-NonCommercial 4.0 International License (http://creativecommons.org/licenses/by-nc/4.0/) which permits copy and redistribute the material just in noncommercial usages, provided the original work is properly cited. 
Most of the reports of abortion from Iran are based on face to face interview $(5,6)$, which are affected by underreporting (2). In a household survey conducted in Tehran, the capital of Iran, 2470 women in childbearing age were interviewed, of whom $45.7 \%$ reported at least one lifetime abortion (6). Nearly one-fifth of these women reported the induced abortion in their lifetime (6). In another study conducted in Northwest Iran, $17 \%$ of the interviewees had been experienced at least one episode of induced abortion $(7,8)$.

No authority has recommended that the direct interview is as a reliable method of estimating abortion rate, especially in settings in which abortion highly prohibited due to religious and legal barriers (2). Because of these barriers, the application of the direct methods for estimating the frequency of abortion is very limited. Most abortions are not being registered in medical records. In addition, direct questioning from women is prone to different biases. Furthermore, it is very difficult to take a very large random sample from the whole community, particularly from marginalized women who may have much more abortions, such as female sex workers. On top of that, because of culture, legal and religious barriers, women usually do not disclose their abortions, particularly illegal ones.

Hence, indirect techniques for estimating the size of abortion, such as Network Scale-up (NSU), might be good alternative solutions. In NSU, people reply about the frequency of a characteristic among their personal networks (9). Since, this frequency has a direct association with the frequency of that characteristic in the community, we may address to the question of interest indirectly. In other words, people do not reply to the sensitive question, such as the history of abortion, about themselves but also they reply on behalf of the persons in their networks. Based on the above explanation, we used NSU in national level to assess the frequency of abortions with and without medical indications (AWMI+ and AWMI-) among Iranian women in the last year.

\section{Objectives}

The aim of this study was to explore the practicality of an indirect method of estimating the rate of abortion, known as Network Scale-up, and to provide the estimation about the episode of abortion with and without medical indications (AWMI+ and AWMI-) in Iran.

\section{Materials and Methods}

Iran, as a developing country in Middle East with an Islamic culture, has 75 million populations, of which 22390943 are women aged 15 and 49 years. This was a cross-sectional study that conducted in 2012. In this study, we selected a random sample from urban people more than 18 years old using the multistage sampling technique. A sample between 200 and 1000 persons was selected from each province of Iran (31 provinces). Within each province, around $75 \%$ of the samples were selected from the capital, and the remaining from one or two large cities. For the sake of comprehensibility by lay persons, we adopted "non-medical" abortion instead of using "illegal" abortion (4).

We stratified each city to three zones based on the social and economic classes. In each stratum, two to four random streets were selected. Only pedestrians who walked alone were approached. We tried to recruit pedestrians from different age and sex groups to get a representative sample. Samples were approached in streets, and the interviewer explained the main objectives of the study and encouraged the pedestrians to cooperate. The street-based sampling was selected based on the results of a methodological study, which showed that Iranian people reply to sensitive questions more accurately, since they were more confident that they would not be tracked comparing to the household or telephone-based sampling (comparison of the three interview methods on response pattern to the sensitive and non-sensitive questions [accepted for publication]). Only pedestrians who were walking alone were approached by a same sex interviewer. To get a more representative sample, a maximum variation rule was used to recruit samples with maximum heterogeneity in their demographic variables ( e.g. clothing, age, gender, education level, etc.). The objectives of the study were explained to pedestrians and only those consented to participate were approached. For further exploration, demographic characteristics of the respondents were recorded. We ensured the respondents about the anonymity and confidentiality of their answers.

\subsection{NSU Methodology}

The assumption behind NSU is that the overall people's network (shown by $\mathrm{C}$ ) represents the target population fairly. Here we simply asked respondents how many people they know in each of hidden groups (shown by m) (9). The proportion of hidden groups in the respondents' network should be scaled up to get the final size in the whole community (shown by e). Here e stands for the size of community, i and j represent the respondent and hidden population of interest, respectively (9-12):

According to the usual practice, definition of 'know' was mutual recognition of each other by sign or name, may be contacted, and in the past two years have had contact either in person, face-to-face, phone or by email $(9,13$, 14). As noted earlier, the NSU requires the estimation of average network size of the population (shown by C). We calculated the average network size of Iranian at 308 (15).

One potential limitation of the NSU studies is the transmission error. It means that some of the members of a respondent's network might have had abortion, but he/she might not be aware of that. For example, the transparency rate of $50 \%$ means that in average people are aware of half of abortions in their personal network. Therefore, in order to verify the NSU estimations, we have to divide the fi- 
nal estimate by the visibility factor. To estimate the visibility factor, we approached 34 midwifes and gynecologists in different parts of Iran. We explained the concept of 'transparency' and asked them about the minimum and maximum transparency factors of AWMI+ and AOMI-. The average of their responses was applied as the visibility factors. Clearly, the lower the visibility factor, the higher the final estimate. In addition, negative binomial regression model was fitted to investigate the impact of demographic characteristics of the respondents on their response.

To provide the uncertainty level for estimates, we applied the Monte Carlo technique. We allowed for uncertainty in peoples' reply ( $\mathrm{m}$ ) and visibility factor. Random numbers from the normal and uniform distributions were generated 1000 times, respectively. Percentiles of 2.5 and 97.5 were considered as the lower and upper bounds of the uncertainty limit. We performed our analysis at national level. However, we mapped the rates of abortions in provinces using the GIS technique. For this analysis, using Bayesian concepts, we adjusted the observed rates of every province based on the national rate of abortion as prior probability. Provinces were classified into three levels based on percentiles. These computations were done using SPSS version 20 and Microsoft Excel 2010 software. The maps were created using Arc Map version 9.3 Software. This work has been granted by Ministry of Health and Medical Education. We explained the aim of the study and only those who verbally agreed to fill the questionnaires were recruited. The code of ethical approval was 163/90/KA.

\section{Results}

We recruited 12960 subjects; among them 112 cases had the unreliable responses (i.e. above 100). We removed such responses in accordance with the usual practice. Our final sample comprised of $52 \%$ male, the mean (standard deviation) age values for male and female samples were 32.5 (11.5) and 32.9 (12.2), respectively; the youngest and oldest subjects were 18 and 87 years old, respectively. Nearly $47 \%$ of the respondents aged more than 30 years, which was fairly close to the proportion of this age group in the country (43\%). About $37 \%$ of the samples were single, and around $43 \%$ of the respondents had an academic degree. We compared age and sex distribution of our sample with that of general population and no significant difference was seen.

Around $71 \%$ of the respondents did not know any women who had abortion in the last year. Mean \pm SD of the number of abortions known was $0.47 \pm 0.98$; however, the average number of total abortions reported by male respondents was lower than female (0.33 vs. 0.62 ), and male were $45 \%$ less likely to know abortions in the last year (Table 1). In addition, respondents aged 18 to 30 were $12 \%$ more likely to know women who had abortion. Education was positively associated with the number of abortions known. In addition, the married respondents compared to single subjects were $28 \%$ more likely to know a woman who had abortion in the last year. Corresponding figure for the divorced and married subjects compared to single respondents, was $31 \%$.

\subsection{Abortion With Medical Indications (AWMI+)}

Regarding AWMI+, we applied the minimum and maximum visibility factors at 0.43 and 0.75 , respectively. Applying the maximum visibility factor at 0.75 , our minimum estimation for the annual number of abortions, was 95257 (95\% C.I. $84,558,109,653$ ). This was corresponded to 4.67 abortions in 1000 women aged 15 to 44 years old, and 70.54 abortions in 1000 pregnancies. When we applied the minimum transparency at 0.43 , the maximum estimate for the annual number of abortions reached 166146 , corresponded to 8.15 abortions out of 1000 women aged 15-44 years, or 116.9 abortions per 1000 pregnancies.

\begin{tabular}{|c|c|c|c|c|c|}
\hline Variable & AWMI+ & AWMI- & Total Abortion & IRR (95\% C.I) & PValue \\
\hline Sex & & & & & $<0.001$ \\
\hline Male & $0.20 \pm 0.60$ & $0.13 \pm 0.51$ & $0.33 \pm 0.82$ & $0.55(0.51,0.58)$ & \\
\hline Female & $0.39 \pm 0.85$ & $0.23 \pm 0.70$ & $0.62 \pm 1.12$ & REF & \\
\hline Age & & & & & 0.004 \\
\hline $18-30, y$ & $0.28 \pm 0.72$ & $0.19 \pm 0.64$ & $0.47 \pm 0.98$ & $1.12(1.04,1.20)$ & \\
\hline$>30, y$ & $0.31 \pm 0.75$ & $0.17 \pm 0.58$ & $0.48 \pm 0.99$ & REF & \\
\hline \multicolumn{6}{|l|}{ Marriage status } \\
\hline Married & $0.33 \pm 0.78$ & $0.18 \pm 0.59$ & $0.51 \pm 1.01$ & $0.66(1.18,1.38)$ & 0.006 \\
\hline Divorce/Widow & $0.26 \pm 0.77$ & $0.25 \pm 0.72$ & $0.51 \pm 1.04$ & $0.75(1.08,1.59)$ & $<0.001$ \\
\hline Single & $0.24 \pm 0.65$ & $0.18 \pm 0.63$ & $0.42 \pm 0.93$ & REF & \\
\hline \multicolumn{6}{|l|}{ Education } \\
\hline$<12, y$ & $0.26 \pm 0.66$ & $0.16 \pm 0.57$ & $0.43 \pm 0.90$ & $1.28(0.52,0.68)$ & $<0.001$ \\
\hline $12-16, y$ & $0.32 \pm 0.79$ & $0.20 \pm 0.64$ & $0.52 \pm 1.05$ & $1.31(0.65,0.86)$ & $<0.001$ \\
\hline$>16, y$ & $0.43 \pm 0.99$ & $0.24 \pm 0.67$ & $0.66 \pm 1.29$ & REF & \\
\hline
\end{tabular}

\footnotetext{
a Abbreviation: IRR: Incidence Rate Ratio, AWMI+: abortions with medical indication, AWMI-: abortions without medical indication, SD: standard deviation.

$\mathrm{b}$ Data presented as mean $\pm \mathrm{SD}$.
} 
Rastegari A et al.

Table 2. Estimation of the Number of Medical and non-Medical Abortions in the Last Year, Based on Minimum and Maximum Transparency Level

\begin{tabular}{|c|c|c|c|c|}
\hline \multirow[t]{2}{*}{ Variable } & \multicolumn{2}{|c|}{ Abortion With Medical Indications (AWMI +) } & \multicolumn{2}{|c|}{ Abortion Without Medical Indications (AWMI-) } \\
\hline & $\begin{array}{c}\text { Maximum estimate } \\
\text { (Minimum transparency) }\end{array}$ & $\begin{array}{c}\text { Minimum estimate } \\
\text { (Maximum transparency) }\end{array}$ & $\begin{array}{c}\text { Maximum estimate } \\
\text { (Minimum transparency) }\end{array}$ & $\begin{array}{l}\text { Minimum estimate } \\
\text { (Maximum transparency) }\end{array}$ \\
\hline Transparency factor & 0.43 & 0.75 & 0.20 & 0.34 \\
\hline $\begin{array}{l}\text { The total number of } \\
\text { abortions ( } 95 \% \text { levels } \\
\text { of uncertainty) }\end{array}$ & $166146(131089,212624)$ & $95257(84558,109653)$ & $219248(176673,288538)$ & $128969(113261,150068)$ \\
\hline $\begin{array}{l}\text { Abortion rate per } \\
1000 \text { women aged } \\
15-44 \text { years }\end{array}$ & 8.15 & 4.67 & 10.75 & 6.33 \\
\hline $\begin{array}{l}\text { Abortion rate per } \\
1000 \text { pregnancies }\end{array}$ & 116.9 & 70.54 & 148.7 & 93.18 \\
\hline
\end{tabular}

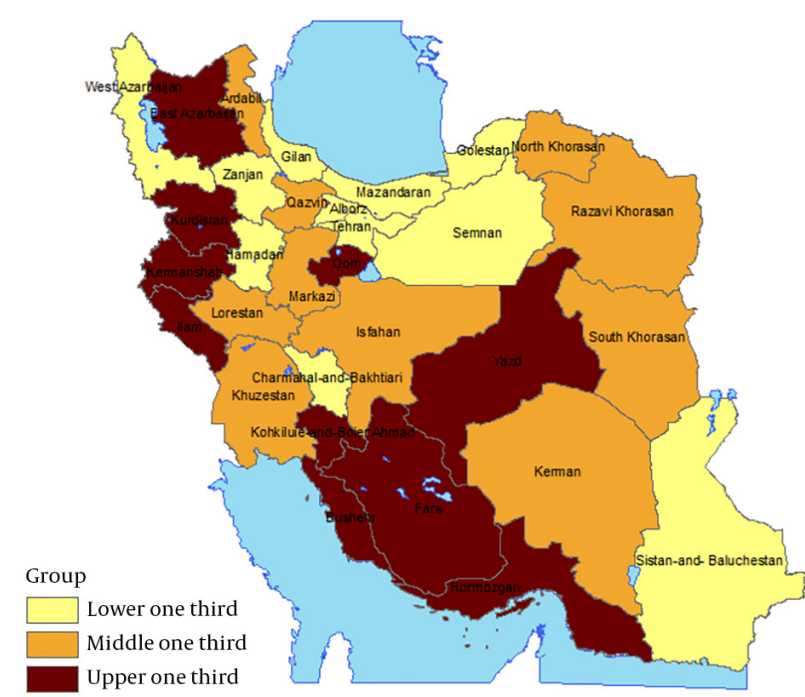

Figure 1. Distribution of Abortions With Medical Indications in the Last Year

\subsection{Abortion Without Medical Indications (AWMI-)}

The minimum and maximum visibility factors applied to estimate AWMI- were 0.20 and 0.34 , respectively. Applying the maximum visibility factor, the minimum number of last year abortions was estimated at 128969 (95\% C.I. 113 261, 150 068) (Table 2). This was corresponded to 6.33 abortions among 1000 women (or 93.18 abortions out of 1000 pregnancies). Under the minimum visibility factor, the maximum number of last year abortions was 219248 (95\% C.I. 176 673, 288 538). This indicates 10.75 abortions among 1000 women, or 148.7 abortions in 1000 pregnancies.

Figure 1 and 2 demonstrate the geographical variation of AWMI+ and AWMI-, and the total number of abortions

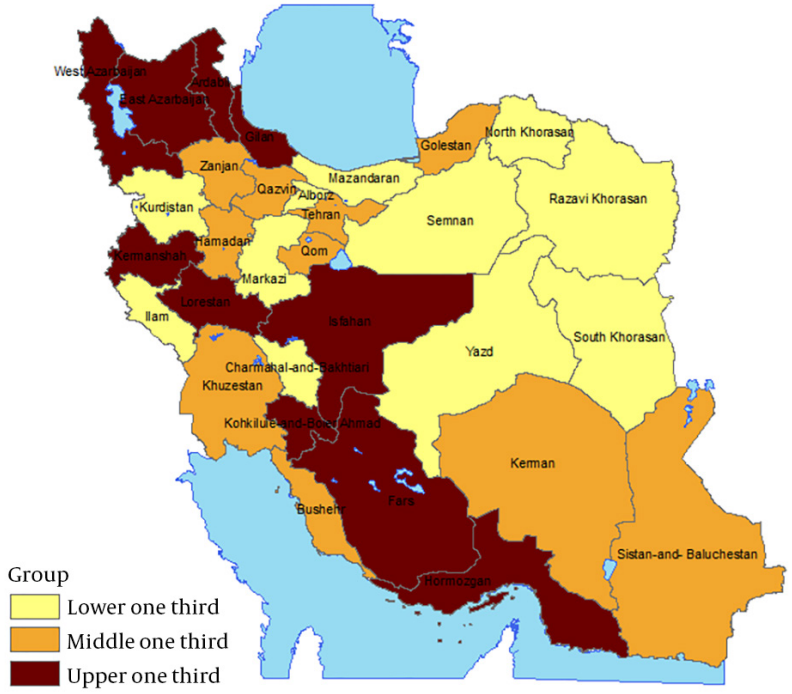

Figure 2. Distribution of Abortions Without Medical Indications in the Last Year

at provincial level. The correlation coefficient between the frequencies of AWMI+ and AWMI- abortions was 0.50. This indicates that, at provincial level, the higher the AWMI+, the higher the AWMI-. We found two clusters of provinces with the highest rate of both AWMI+ and AWMI- in west and northwest, and in south and center of Iran. On the other hand, a clear cluster of low AWMI- was observed in north-east of Iran. As a sensitivity analysis, different visibility factors were applied and the AWMI+ and AWMI- were estimated. Figure 3 demonstrates the variability of our estimates in the range of visibility factors applied. It can be seen that the lower the visibility, the higher the final estimate. 
Rastegari A et al.

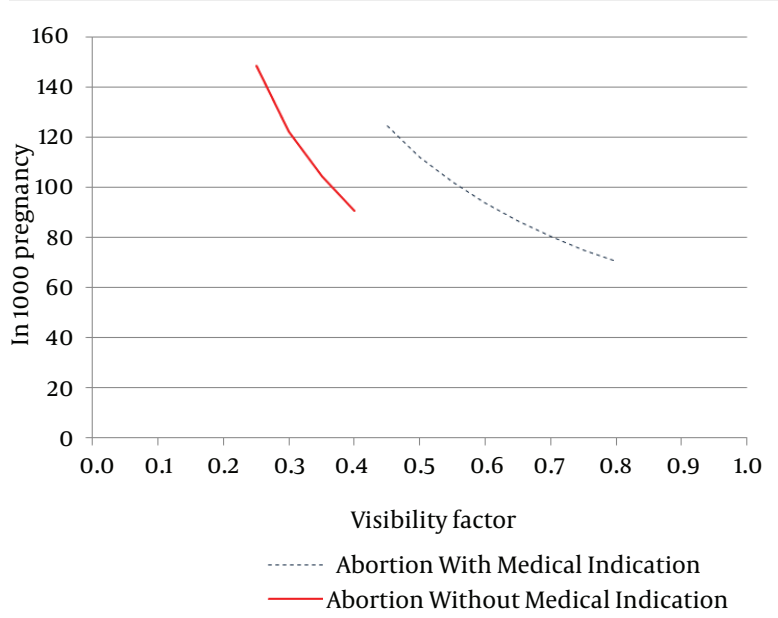

Figure 3. Sensitivity Analysis of the Estimated Number of Abortions Applying Different Visibility Factors

\section{Discussion}

This study applies the NSU, an indirect size estimation approach, to estimate the annual number of AWMI+ and AWMI- in Iran. About 30\% of the respondents were aware of one person who had abortion in the previous year. Considering the private nature of abortion in Iran the figure warns us that abortion is a "common practice" in Iran (16). Abortion, in both medical and nonmedical cases will bring different health hazards to maternal and family health. Abortion- related problems are not limited to the physical health. It has different mental health consequences including depression, anxiety, suicide efforts and even drug abuse by parents (17). Abortion is one of the most important factors contributing maternal mortality and morbidity as well. According to the international data, $13 \%$ of the maternal deaths occur after unsafe abortions (18). Abortion is considered as an illegal act in Iran. Since most women who choose to have an abortion attempt to conceal it, it occurs in an unsafe situation which potentially increases the health hazards. As it was expected male were less likely to know someone who practiced abortion in the past year, since in Iranian culture, females may feel a greater sense of shame when discussing about abortion with a male than female. The number of abortions known was reported more by the younger subjects than those older than 30 years. In an interview survey conducted in Tehran, the capital of Iran, the largest proportion of induced abortion was reported by those in age range of 15 to 24 years old $(2,4,5,7)$. The abortion rates were different throughout the country. Based on the current survey Hormozgan, Fars (in south), and Gilan (in north) were amongst the provinces with the highest rate of AWMI-, which falls in line with the results of Erfani (1). Based on 2000 Iran Demographic and Health Survey, he found that the three aforementioned provinces showed the highest rates of the induced abor- tion (1). Based on our findings, there are large variations in the rates of both medical and nonmedical abortions among the provinces. This variation may highlight the need for more deep studies to find the source of such a spatial variability, and local interventions. The annual abortion rate for women aged 15 to 44 was estimated to be between 6.3 and 10.8 per 1000 pregnancies. Extrapolating from the DHS data, Erfani et al. estimated that the corresponding figure was 7.5 per 1000 pregnancies, nationally (2), and in Tehran it has been estimated to be about 6 per 1000 pregnancies (5). Based on the current survey, the annual number of nonmedical abortions ranges between 128969 and 219 248. Different studies report different frequencies of illegal abortion in Iran. In a review that portraits the situation of abortion in countries of the Middle East and North Africa (MENA) regions, the author reports that Iran has had the highest frequency of unsafe abortions compared to the other MENA countries for the period 1995-2000 (6). In this report, it has been stated that "over 1000 unsafe abortions take place every day in Iran" (6). In a study in which 2705 women were interviewed, the respondents claimed that $9.3 \%$ of pregnancies terminated in illegal abortion (7). According to the World Fertility Data 2012, about 1300000 births occur in Iran each year so; considering 9.3\% we can expect that at least 120000 illegal abortions occur annually. In interpreting the figures obtained from the interview data the high probability of underreporting should be put in mind. In other words, the annual rate of 120000 illegal abortions per year seems to be less than real. Comparing to DHS-based data $(2,3)$ and interview surveys $(1,8)$ we can conclude that NSU yield a higher frequency of illegal abortion. It has been proposed that the difference between rates of abortion among countries is influenced by three factors; contraceptive prevalence and effectiveness, fertility desire, and the probability of aborting unintended pregnancies (19). In Iran, a high proportion of couples use traditional methods (i.e. 22.3\%) and about 30\% of pregnancies are unintended (20). Although we have no accurate data on the probability of recourse to abortion in the country, considering the fact that $58 \%$ of the unintended pregnancies end up in abortion, worldwide (19), it seems that all three factors are acting together to develop an abortion syndetic.

\subsection{Strengths and Weaknesses of the Current Study}

Subject sensitivity according to the privacy and its legal aspects is one of the limitations of the study. In some cases, some pregnant women face the spontaneous abortion and considering the early stages of the pregnancy, they won't be able to recognize it as an abortion; it could play its role as one of the limitations. Small sample size at the provincial level is a limitation to use the results as a reliable statistics. We tried to implement a proportional to size strategy. We assumed that, roughly speaking, at each province $75 \%$ of people live in the capital. Therefore, we 
selected $75 \%$ from capital and $25 \%$ from one main city. It was hugely difficult to manage more than 2 cities at each province. Therefore, our results cannot be generalized to rural population. Finally, gathering information considering 15-49 years old will cause to neglect the abortions beyond these age groups in the programming. The NSU has been designed to assess size of hidden groups. In NSU method, respondents reply on behalf of their network, in the case of non-hidden groups, application of direct methods in simple. Therefore, to the best of our knowledge, no study used NSU to estimate the size of the nonhidden groups. Nevertheless, in order to estimate the network size of people, the size of some non-sensitive reference groups (with known sizes) are being back-calculated. In these exercises the estimations are usually more or less acceptable (15). Our team currently conducted some studies to assess practicality of this method in estimation of size of people with different types of disability. Our primary estimates confirm usefulness of the NSU method. However, results are not published yet. The large sample size, indirect question (asking question through the people social network) and nationwide sampling are the most important strengths of the current study. One more promising issue is the consistency of our findings with other studies.

\subsection{Comments to the Policy Makers}

Abortion is one of the most important causes for maternal mortality and morbidity. Due to this importance, the improvement and strengthening of the reproductive health programs should be considered. It should be settled as a program priority and needs a comprehensive and multi- aspect approach for interventions. To prepare a comprehensive program, having complete and reliable information which approaches the issue through different health, social, and cultural aspects is obligatory. Available researches shows very different ranges for abortions and in some cases cannot explain the main causes for abortion. It is suggested through review all available data, at first the data shortage should be fulfilled, then the complementary researches should be performed and finally the comprehensive intervention abortion reduction program should be prepared through the collaboration with all stakeholders. The study shows the spectrum and depth of abortion practice in Iran. It discussed strongly about the hidden nature of abortion, considering both social and legal reasons. Moreover, it shows the need for an urgent action to prepare a holistic program, approaching the problem through different aspects.

\section{Acknowledgements}

The authors would like to thank the Ministry of Health and Medical Education, Tehran, Iran.

\section{Authors' Contributions}

Aliakbar Haghdoost and Mohammad Reza Baneshi developed the original idea and the protocol. Aliakbar Haghdoost, Mohammad Reza Baneshi, Azam Rastegari, and Saiedeh Haji-maghsoudi contributed in the study design, data acquisition, analysis and interpretation of the data, and writing of the manuscript. Mohammad Eslami and Nowzar Nakhaee helped in writing the analysis interpretation and also in critical revision of the manuscript. Hossein Malekafzali reviewed the manuscript critically and supervised the study.

\section{Financial Disclosure}

All of the authors are independent researchers without any conflict of interest except Dr. Islami who is working in Office of Family Planning in Ministry of Health and Medical Education. He indirectly involves with the concept of abortion. All authors have read and approved the final manuscript.

\section{Funding/Support}

This work was supported by the Ministry of Health and Medical Education, Tehran, Iran.

\section{References}

1. Erfani A. What Do We Know? PSC Dis J. 2008;22(1):1.

2. Erfani A, McQuillan K. Rates of induced abortion in Iran: the roles of contraceptive use and religiosity. Stud Fam Plann. 2008;39(2):111-22.

3. Shah I, Ahman E. Unsafe abortion in 2008: global and regional levels and trends. Reprod Health Matters. 2010;18(36):90-101.

4. Hourieh SM, Abolghasem P, Feizollah A. Knowledge and Attitudes of a Number of Iranian Policy-makers towards Abortion. J Reprod Infertil. 2010;11(3):189-95.

5. Erfani A. Induced abortion in Tehran, Iran: estimated rates and correlates. Int Perspect Sex Reprod Health. 2011;37(3):134-42.

6. Hessini L.Abortion and Islam: policies and practice in the Middle East and North Africa. Reprod Health Matters. 2007;15(29):75-84

7. Ranji A. Induced abortion in Iran: prevalence, reasons, and consequences. J Midwifery Womens Health. 2012;57(5):482-8.

8. Shapiro GK. Abortion law in Muslim-majority countries: an overview of the Islamic discourse with policy implications. Health Policy Plan. 2014;29(4):483-94.

9. Paniotto V, Petrenko T, Kupriyanov O, Pakhok O. Estimating the size of populations with high risk for HIV using the network scale-up method. Kiev Inter Inst Soci. 2009.

10. Killworth PD, McCarty C, Bernard HR, Shelley GA, Johnsen EC. Estimation of seroprevalence, rape, and homelessness in the United States using a social network approach. Eval Rev. 1998;22(2):289-308.

11. Salganik MJ, Mello MB, Abdo AH, Bertoni N, Fazito D, Bastos FI The Game of Contacts: Estimating the Social Visibility of Groups. Soc Networks. 2011;33(1):70-8.

12. Shokoohi M, Baneshi MR, Haghdoost AA. Size Estimation of Groups at High Risk of HIV/AIDS using Network Scale Up in Kerman, Iran. Int J Prev Med. 2012;3(7):471-6.

13. Midanik LT, Greenfield TK. Telephone versus in-person interviews for alcohol use: results of the 2000 National Alcohol Survey. Drug Alcohol Depend. 2003;72(3):209-14.

14. Pridemore WA, Damphousse KR, Moore RK. Obtaining sensitive information from a wary population: a comparison of telephone and face-to-face surveys of welfare recipients in the United States. Soc Sci Med. 2005;61(5):976-84.

15. Rastegari A, Haji-Maghsoudi S, Haghdoost A, Shatti M, Tarjoman T, Baneshi MR. The estimation of active social network size of the Iranian population. Glob J Health Sci. 2013;5(4):217-27. 


\section{Rastegari A et al.}

16. Nojomi M, Akbarian A, Ashory-Moghadam S. Burden of abortion: induced and spontaneous. Arch Iran Med. 2006;9(1):39-45.

17. Fergusson DM, Horwood LJ, Ridder EM. Abortion in young women and subsequent mental health. J Child Psychol Psychiatry. 2006;47(1):16-24.

18. Azmat SK, Bilgrami M, Shaikh BT, Mustafa G, Hameed W. Perceptions, interpretations and implications of abortions: a qualita- tive enquiry among the legal community of Pakistan. Eur J Contracept Reprod Health Care. 2012;17(2):155-63.

19. Bongaarts J, Westoff CF. The potential role of contraception in reducing abortion. Stud Fam Plann. 2000;31(3):193-202.

20. Motlaq ME, Eslami M, Yazdanpanah M, Nakhaee N. Contraceptive use and unmet need for family planning in Iran. Int J Gynaecol Obstet. 2013;121(2):157-61. 